ACCepted for publichtion in the Astrophysical Journal Letters

Preprint typeset using LATEX style emulateapj v. 12/16/11

\title{
FIRST CHARACTERIZATION OF THE NEUTRAL ISM IN TWO LOCAL VOLUME DWARF GALAXIES
}

\author{
Lilly Bralts-Kelly, Alyssa M. Bulatek, Sarah Chinski, Robert N. Ford, Hannah E. Gilbonio, Greta Helmel, \\ Riley McGlasson, Andrew Mizener, John M. Cannon \\ Department of Physics \& Astronomy, Macalester College, 1600 Grand Avenue, Saint Paul, MN 55105, USA
}

Serafim Kaisin, Igor Karachentsey

Special Astrophysical Observatory of RAS, Nizhnij Arkhyz, KChR, 369167, Russia

\section{GRANT DENN}

Department of Physics, Metropolitan State University of Denver, P.O. Box 173362, Denver, CO 80217, USA Accepted for publication in the Astrophysical Journal Letters

\begin{abstract}
We present the first HI spectral line images of the nearby, star-forming dwarf galaxies UGC 11411 and UGC 8245, acquired as part of the "Observing for University Classes" program with the Karl G. Jansky Very Large Array (VLA kinematics of each system. Comparing with HST broadband and ground-based $\mathrm{H} \alpha$ imaging, we find that the ongoing star formation in each galaxy is associated with the highest HI mass surface density regions. UGC 8245 has a much lower current star formation rate than UGC 11411, which harbors very high surface brightness $\mathrm{H} \alpha$ emission in the inner disk and diffuse, lower surface brightness nebular gas that extends well beyond the stellar disk as traced by HST. We measure the dynamical masses of each galaxy and find that the halo of UGC 11411 is more than an order of magnitude more massive than the halo of UGC 8245, even though the HI and stellar masses of the sources are similar. We show that UGC 8245 shares similar physical properties with other well-studied low-mass galaxies, while UGC 11411 is more highly dark matter dominated. Both systems have negative peculiar velocities that are associated with a coherent flow of nearby galaxies at high supergalactic latitude.
\end{abstract}

Keywords: galaxies: evolution - galaxies: dwarf — galaxies: irregular — galaxies: individual (UGC 11411, UGC 8245)

\section{INTRODUCTION}

Nearby dwarf galaxies provide unique insights into the physical properties of low-mass halos. Those systems which can be resolved into individual stars allow the complex relationships between active star formation and the surrounding interstellar medium (ISM) to be examined in detail. Similarly, in those systems where the bulk rotation velocity can be extracted, the distributions of dark and baryonic matter can be examined on a spatially resolved basis.

Major single-dish HI surveys have now cataloged thousands of gas-rich objects in the local volume (e.g., HIPASS - Meyer et al. 2004; ALFALFA - Giovanelli et al. 2005). From the spatially resolved perspective, multiple recent and ongoing HI surveys have studied the physical properties of many low-mass galaxies in the local volume (e.g., Swaters et al. 2002; Begum et al. 2008; Cannon et al. 2011; Ott et al. 2012; Hunter et al. 2012; Kirby et al. 2012; Lelli et al. 2014; Wang et al. 2017). However, each HI survey has its own selection criteria, and therefore not all local dwarf galaxies have been examined in the HI spectral line on a spatially resolved basis. Some star-forming, gas-rich dwarf galaxies possess

jcannon@macalester.edu

ikar@sao.ru, skai@sao.ru

gdenn@msudenver.edu

a 'The National Radio Astronomy Observatory is a facility of the National Science Foundation operated under cooperative agreement by Associated Universities, Inc. extensive observations at various wavelengths but have yet to have their neutral ISM examined in detail. In this manuscript, we present the first observations of the HI $21 \mathrm{~cm}$ emission line in two such galaxies: UGC 11411 and UGC 8245 (see Table1for a summary of physical properties). Note that each galaxy has a very low (barycentric) recessional velocity; the proximity of Milky Way foreground HI gas may have precluded detailed HI studies to date.

These galaxies were selected for study based on simple selection criteria: each hosts ongoing star formation and has a direct distance measurement from resolved Hubble Space Telescope (HST) photometry that places the galaxy within the local volume. Further, to date, no measurements (single-dish or interferometric) of their HI gas contents have been published. These systems thus increase the number of nearby dwarf galaxies with detailed observations of the neutral gas component.

\section{OBSERVATIONS AND DATA HANDLING}

HI spectral-line observations of UGC11411 and UGC 8245 were acquired with the VLA in the D configuration in February and March of 2017 for programs TDEM0023 and TDEM0024, respectively. The data sets are part of the "Observing for University Classes" program, a service provided by NRAO as an opportunity for courses teaching radio astronomy theory to acquire new observations to be analyzed by students (Cannon \& Van Moorsel 2017). 
Data handling and imaging followed the prescriptions described in McNichols et al. (2016) and Teich et al. (2016). Briefly, a $4.0 \mathrm{MHz}$ bandwidth is separated into 1024 channels, delivering a native spectral resolution of $0.86 \mathrm{~km} \mathrm{~s}^{-1} \mathrm{ch}^{-1}$. The primary calibrator was $3 \mathrm{C} 286$; the phase calibrators were $\mathrm{J} 1748+7005$ and $\mathrm{J} 1323+7942$ for UGC 11411 and UGC 8245, respectively. The total on-source integration time was $\sim 1.5$ hours for each source. The VLA data were calibrated using standard prescriptions in the CASA 1 environment. Continuum subtraction was performed in the $u v$ plane using a firstorder fit to line-free channels bracketing the galaxy in the central $50 \%$ of the bandpass.

Each dataset was imaged via the CASA task CLEAN with $10^{\prime \prime}$ pixels at $2 \mathrm{~km} \mathrm{~s}^{-1}$ velocity resolution. Cleaning was performed to 2.5 times the rms noise per channel in line-free channels. The synthesized beam sizes and rms noises are $61.68^{\prime \prime} \times 44.30^{\prime \prime}$ and $3.3 \mathrm{mJy} \mathrm{Bm}^{-1}$ (UGC 11411) and $60.58^{\prime \prime} \times 40.04^{\prime \prime}$ and $3.0 \mathrm{mJy} \mathrm{Bm}^{-1}$ (UGC 8245). The resulting cubes were threshold blanked at the $2.5 \sigma$ level and then further blanked by hand to isolate emission that is coherent in velocity space. Finally, the cubes were collapsed to create traditional moment maps representing HI mass surface density and intensity weighted velocity field.

Both target galaxies have low recessional velocities (see Table 1 and further discussion in $\S$ 4). We thus pay particular attention to the possibility of contamination of each line profile by Milky Way foreground HI gas. For UGC 11411 the emission from the galaxy is cleanly separated in velocity space from Milky Way gas; the lowest velocity HI gas detected from the source is located at $+35 \mathrm{~km} \mathrm{~s}^{-1}$. For UGC 8245, however, there is moderate contamination by Milky Way gas; specifically, in the velocity range of $-10 \mathrm{~km} \mathrm{~s}^{-1}$ to $+6 \mathrm{~km} \mathrm{~s}^{-1}$, there is extended foreground gas detected over much larger angular scales than the galaxy. At these velocities we are able to spatially differentiate this extended gas from the comparatively compact emission from the target galaxy. We note that at these velocities, the absolute flux from UGC 8245 is less certain than at the more negative velocities where the galaxy is cleanly separated from the Milky Way foreground. Based on the symmetry of the HI gas about the systemic velocity (see Section $\S$ 3), the contamination appears to be a minor effect.

We compare our new HI imaging with archival groundbased and HST optical imaging. The optical imaging was acquired with the SAO $6.0 \mathrm{~m}$ telescope; detailed descriptions of data handling can be found in Karachentsev \& Kaisina (2013). The flux-calibrated and continuumsubtracted $\mathrm{H} \alpha$ images were registered to the WCS coordinate system of the HST images to create the images presented in this work.

\section{ANALYSIS}

\subsection{The Neutral ISM of UGC11411 and UGC 8245}

HI images of UGC 11411 and UGC 8245 are presented in Figures 1 and 2. respectively. While the angular resolution is coarse, the galaxies are formally resolved, both along the major and the minor axes of the beams. The moment zero maps localize the HI gas and provide

\footnotetext{
${ }^{1}$ https://casa.nrao.edu
}

Table 1

Basic Characteristics of UGC 11411 and UGC 8245

\begin{tabular}{lcc}
\hline \hline \multicolumn{1}{c}{ Parameter } & UGC 11411 & UGC 8245 \\
\hline Right ascension (J2000) & $19^{\mathrm{h}} 08^{\mathrm{m}} 42^{\mathrm{s}} 3$ & $13^{\mathrm{h}} 08^{\mathrm{m}} 36 .^{\mathrm{s}} 2$ \\
Declination (J2000) & $+70^{\circ} 17^{\prime} 02^{\prime \prime}$ & $+78^{\circ} 56^{\prime} 14^{\prime \prime}$ \\
Distance $(\mathrm{Mpc})$ & $6.58 \pm 0.12^{\mathrm{a}}$ & $4.72 \pm 0.07^{\mathrm{a}}$ \\
$\mathrm{V}_{\mathrm{HI}}\left(\mathrm{km} \mathrm{s}^{-1}\right)^{\mathrm{b}}$ & $88 \pm 3$ & $-26 \pm 3$ \\
$\mathrm{M}_{\mathrm{B}}(\mathrm{Mag})$ & $-14.01^{\mathrm{c}}$ & $-13.76^{\mathrm{c}}$ \\
$\mathrm{M}_{\star}\left(\mathrm{M}_{\odot}\right)$ & $7.1 \times 10^{7 \mathrm{c}}$ & $7.3 \times 10^{7 \mathrm{~d}}$ \\
$\mathrm{~S}_{\mathrm{HI}}\left(\mathrm{Jy} \mathrm{km} \mathrm{s}^{-1}\right)$ & $3.0 \pm 0.3$ & $2.6 \pm 0.3$ \\
$\mathrm{M}_{\mathrm{HI}}\left(\mathrm{M}_{\odot}\right)$ & $(3.1 \pm 0.46) \times 10^{7}$ & $(1.4 \pm 0.21) \times 10^{7}$ \\
$\mathrm{M}_{\mathrm{dyn}}\left(\mathrm{M}_{\odot}\right)^{\mathrm{e}}$ & $2.3 \times 10^{9}$ & $1.7 \times 10^{8}$ \\
$\mathrm{M}_{\mathrm{dyn}}\left(\mathrm{M}_{\odot}\right)^{\mathrm{f}}$ & $1.4 \times 10^{9}$ & $1.5 \times 10^{8}$ \\
\hline
\end{tabular}

a Tully et al. (2013)

b Systemic velocity based on the new HI images presented in this work.

c Karachentsev et al. (2013)

d Revising the stellar mass from Cook et al. (2014) for the adopted distance of $4.72 \pm 0.07 \mathrm{Mpc}$. This value can be compared to $\mathrm{M}_{\star}=$ $6.0 \times 10^{7} \mathrm{M}_{\odot}$ when applying the same techniques as used to determine the stellar mass of UGC 11411 (Karachentsev et al. 2013).

e Derived using the HST-based inclinations of $i=37^{\circ}$ and $i=75^{\circ}$ for UGC 11411 and UGC 8245, respectively (see $\S 3.2$.

f Derived using the ground-based inclinations of $i=52^{\circ}$ and $i=$ $90^{\circ}$ for UGC 11411 and UGC 8245, respectively (see $\S 3.2$.

high surface brightness sensitivity; the elongation of each galaxy (especially in the directions parallel to the synthesized beam minor axis) is physically meaningful and is not a resolution effect. The HI column densities peak above $5 \times 10^{20} \mathrm{~cm}^{-2}$ in each source; higher angular resolution images are expected to increase the column densities within the inner disk region of each source.

The moment one maps, representing intensityweighted velocity, reveal the bulk projected rotation of each source. While we anticipate that higher angular resolution images will reveal significant kinematic substructure within each galaxy's disk, our low resolution images reveal coherent projected rotation of each galaxy (see also discussion in $\S 3.3$. The intensity weighted velocity fields shown in Figures 1 and 2 are characterized by nearly-parallel isovelocity contours in the inner region of each disk. Importantly, while the northerly declination of each galaxy results in an asymmetric beam shape, there is a fortuitous alignment of the beam minor axis and the kinematic major axis in each galaxy; the signatures of rotation can be interpreted with confidence.

\subsection{Stellar Populations and Star Formation in $U G C 11411$ and $U G C 8245$}

UGC 11411 is an actively star-forming dwarf galaxy. As shown in Figure 3(a), HST observations of UGC 11411 resolve individual stars; this provides a CMD-based distance measurement of $\mathrm{D}=6.58 \pm 0.12 \mathrm{Mpc}$ (Tully et al. 2013). Nebular emission is prominent in the HST images, and numerous stellar clusters and massive star formation complexes are evident. The continuum-subtracted $\mathrm{H} \alpha$ image shown in Figure 3(b) reveals widespread, high surface brightness $\mathrm{H} \alpha$ emission throughout the main body of UGC 11411. Further, there is diffuse $\mathrm{H} \alpha$ nebulosity extending well beyond the stellar disk as traced by HST; note by examining Figure 3(b) that this extended $\mathrm{H} \alpha$ emission is especially prominent in the northwest region. The instantaneous massive star formation rate derived from the $\mathrm{H} \alpha$ image, $\log \left[\mathrm{SFR}_{\mathrm{H} \alpha} /\left(\mathrm{M}_{\odot} \mathrm{yr}^{-1}\right)\right]=-1.84$, 

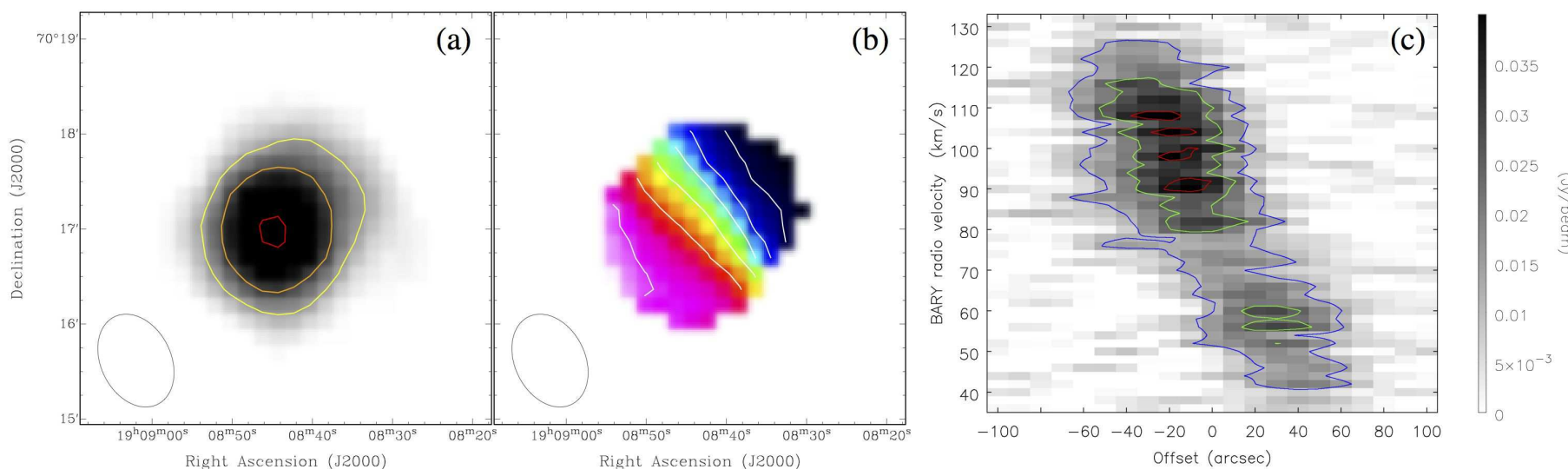

Figure 1. HI images of UGC 11411. Panel (a) shows the HI mass surface density image, with contours overlaid at the $(1.5,3,6) \times 10^{20}$ $\mathrm{cm}^{-2}$ levels, represented by yellow, orange, and red, respectively. Panel (b) shows the intensity weighted HI velocity field, with contours overlaid in $10 \mathrm{~km} \mathrm{~s}^{-1}$ intervals between $56 \mathrm{~km} \mathrm{~s}^{-1}$ and $106 \mathrm{~km} \mathrm{~s}^{-1}$. The beam size is shown in the bottom left of panels (a) and (b). Panel (c) shows a major-axis position-velocity slice. The slice is centered at $(\alpha, \delta)=\left(19^{\mathrm{h}} 08^{\mathrm{m}} 42.5^{\mathrm{s}},+70^{\circ} 17^{\prime} 05.7^{\prime \prime}\right)$, is 5 pixels $\left(50^{\prime \prime}\right)$ wide, and is at a position angle of $+135^{\circ}$ measured east from north. The contours are shown at the $4 \sigma$ (blue), $8 \sigma$ (green), and $12 \sigma$ (red) levels.
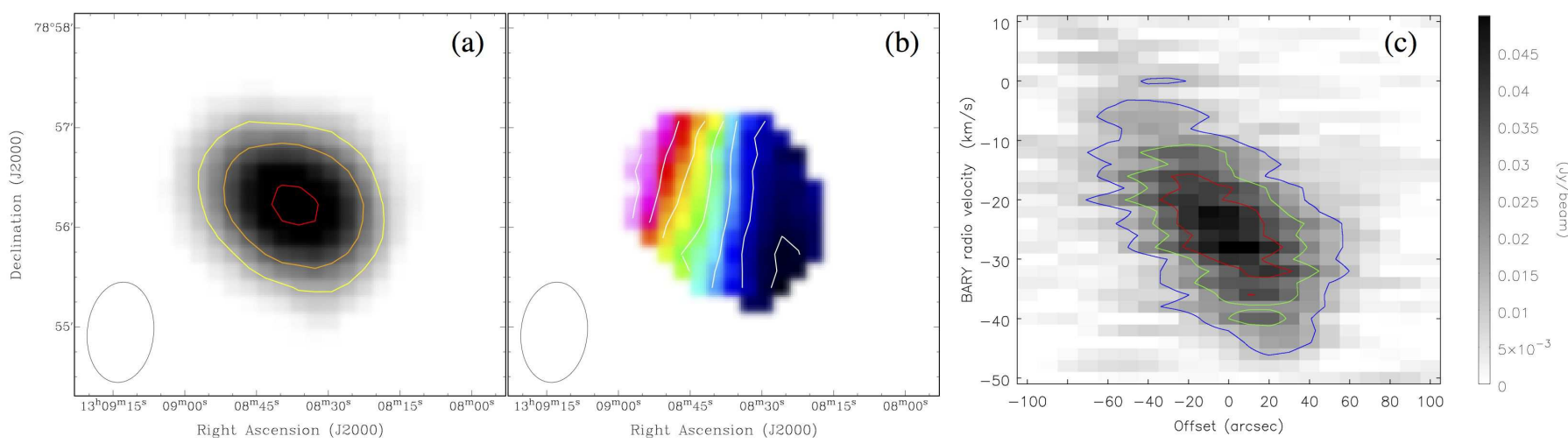

Figure 2. HI images of UGC 8245. Panel (a) shows the HI mass surface density image, with contours overlaid at the $(1.25,2.5,5) \times 10^{20}$ $\mathrm{cm}^{-2}$ levels, represented by yellow, orange, and red, respectively. Panel (b) shows the intensity weighted HI velocity field, with contours overlaid in $3 \mathrm{~km} \mathrm{~s}^{-1}$ intervals between $-31 \mathrm{~km} \mathrm{~s}^{-1}$ and $-13 \mathrm{~km} \mathrm{~s}^{-1}$. The beam size is shown in the bottom left of panels (a) and (b). Panel (c) shows a major-axis position-velocity slice. The slice is centered at $(\alpha, \delta)=\left(13^{\mathrm{h}} 08^{\mathrm{m}} 35.3^{\mathrm{s}},+78^{\circ} 56^{\prime} 14.3^{\prime \prime}\right)$, is 5 pixels $\left(50^{\prime \prime}\right)$ wide, and is at a position angle of $+70^{\circ}$ measured east of north. The contours are shown at the $4 \sigma$ (blue), $8 \sigma$ (green), and $12 \sigma$ (red) levels.

compares well with the longer-timescale, UV-based star formation rate of $\log \left[\mathrm{SFR}_{\mathrm{FUV}} /\left(\mathrm{M}_{\odot} \mathrm{yr}^{-1}\right)\right]=-1.72$ as found by Karachentsev et al. (2013). No constraints on the metallicity of the nebular gas are currently available.

UGC 8245 is a more quiescent system than UGC 11411. As shown in Figure 4. HST observations resolve the stellar population in detail, producing a CMD-based distance $\mathrm{D}=4.72 \pm 0.07 \mathrm{Mpc}$ (Tully et al. 2013). While the stellar population is blue in the inner disk, UGC 8245 does not show any prominent nebular emission in the HST image. The ground-based $\mathrm{H} \alpha$ image reveals two faint star formation complexes; neither is aligned spatially with obvious stellar clusters in the HST images. There is no diffuse or extra-planar $\mathrm{H} \alpha$ emission detected at this sensitivity level. The instantaneous and UV-based star formation rates are lower in UGC 8245 than in UGC 11411: $\log \left[\mathrm{SFR}_{\mathrm{H} \alpha} /\left(\mathrm{M}_{\odot} \mathrm{yr}^{-1}\right)\right]$ $=-3.02$ and $\log \left[\mathrm{SFR}_{\mathrm{FUV}} /\left(\mathrm{M}_{\odot} \mathrm{yr}^{-1}\right)\right]=-2.46$, respectively (Karachentsev \& Kaisina 2013). Interestingly, the brighter HII region on the eastern end of the disk was observed spectroscopically by Berg et al. (2012), resulting in a sub-Solar oxygen abundance measurement ( $\mathrm{Z}$ $\simeq 8-12 \% \mathrm{Z}_{\odot}$ depending on which strong-line method is applied).

A spatially resolved study of the relationships between neutral HI gas and ongoing star formation (as was presented in Teich et al. 2016) is not possible at our present angular resolution. However, comparisons of the locations of the highest $\mathrm{HI}$ mass surface densities and $\mathrm{H} \alpha$ surface brightnesses are possible; Figures $\underline{3}$ (c) and 4(c) show the highest HI column density contour superposed on contours of the $\mathrm{H} \alpha$ emission. In both galaxies, the brightest $\mathrm{H} \alpha$ regions are either co-spatial with, or very close to, the HI maxima. The compact HII regions in UGC 8245 are confined to the gas-rich inner disk. Similarly, in UGC 11411, the major star formation complexes are within or very close to the dense HI gas. However, Figure 3 highlights the remarkable extent of the $\mathrm{H} \alpha$ emission outside of the inner disk.

We use the HST and ground-based images to determine the inclination of each galaxy; a cursory examination of Figures 3 and 4 reveals that UGC 11411 is more face-on than UGC 8245. Following Karachentsev et al. (2013), a first measurement of the inclination is obtained by measuring the apparent axial ratio of each galaxy 


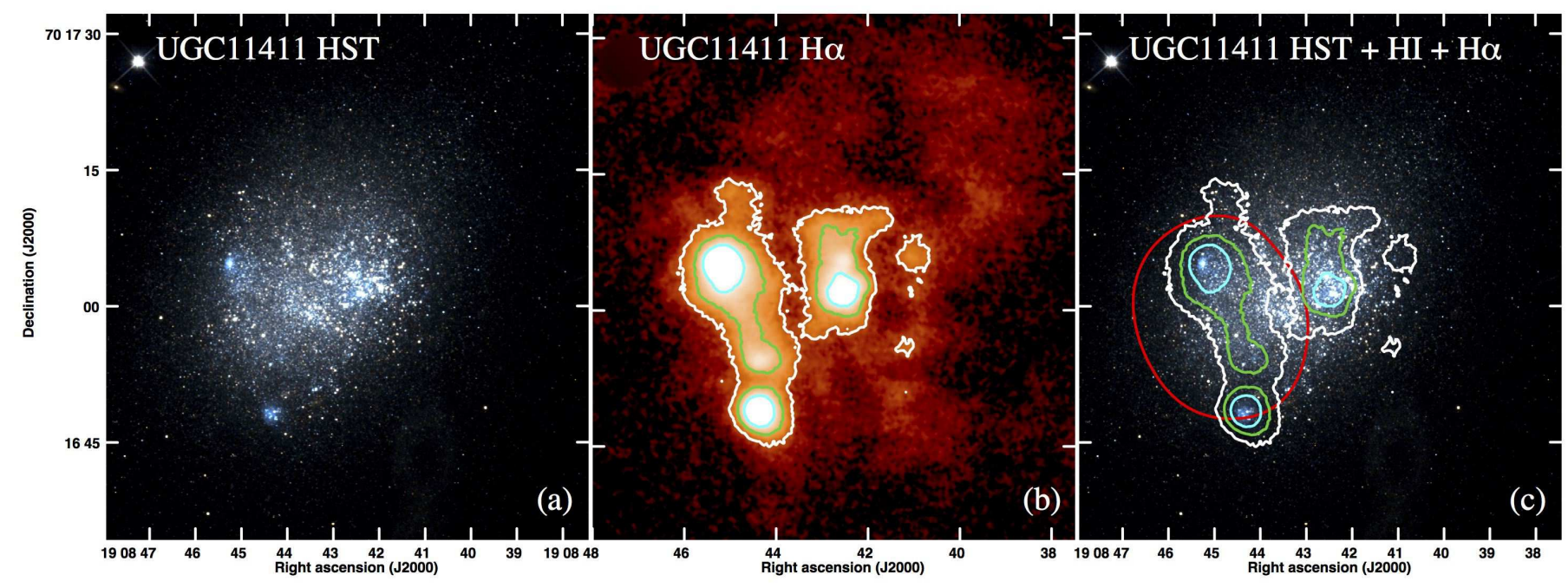

Figure 3. HST 3-color image (a), SAO telescope continuum-subtracted H $\alpha$ image (b), and the HST 3-color image compared with HI and $\mathrm{H} \alpha$ (c) of UGC 11411. The galaxy harbors widespread, high surface brightness $\mathrm{H} \alpha$ emission. The display of the $\mathrm{H} \alpha$ image is logarithmic, with contours separated by 0.4 dex each; note the extended $\mathrm{H} \alpha$ emission to the northwest that is not contoured but is outside of the optical main body. The red contour in panel (c) is the same as shown in Figure $1\left(\mathrm{~N}_{\mathrm{HI}}=6 \times 10^{20} \mathrm{~cm}^{-2}\right.$.)

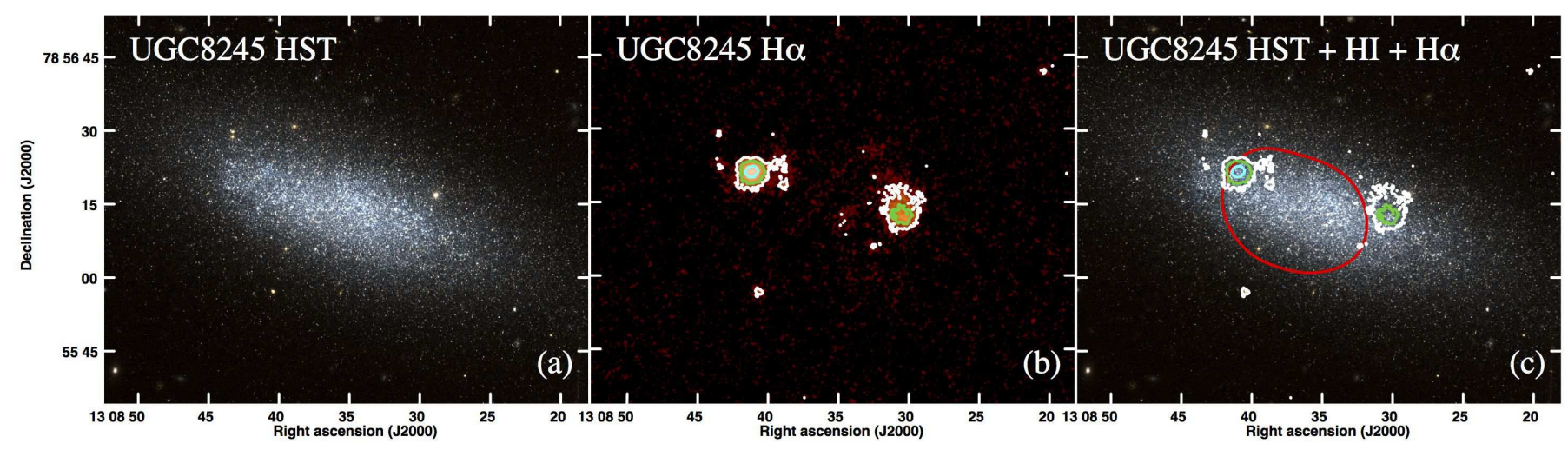

Figure 4. HST 3-color image (a), SAO telescope continuum-subtracted H $\alpha$ image (b), and the HST 3-color image compared with HI and $\mathrm{H} \alpha$ (c) of UGC 8245. The galaxy hosts only two relatively compact and faint HII regions. The display of the H $\alpha$ image is logarithmic, with contours separated by 0.4 dex each. The red contour in panel (c) is the same as shown in Figure $2\left(\mathrm{~N}_{\mathrm{HI}}=5 \times 10^{20} \mathrm{~cm}^{-2}\right.$.)

in the broad-band images that were acquired with the continuum-subtracted $\mathrm{H} \alpha$ images. Assuming an intrinsic axial ratio of 0.42 , we find that $i=52^{\circ}$ and $i=90^{\circ}$ for UGC 11411 and UGC 8245, respectively. This method can be compared with a measurement of the axial ratio of each galaxy in the HST images using the custom software package CleanGalaxy (Hagen et al. 2014). Here, surface brightness contours as a function of galactocentric radius are fit to the HST image after it has been cleaned of foreground and background objects and smoothed with a spatial Gaussian filter. Using this method and assuming the same intrinsic axial ratio of 0.42 , we derive inclinations of $i=37^{\circ}$ and $i=75^{\circ}$ for $\mathrm{UGC} 11411$ and UGC 8245, respectively.

For consistency with the results presented in Teich et al. (2016) and Cannon et al. (2016), we use both values of inclination for each galaxy in the discussion that follows. The lower inclinations dervied using the HST images compared to those using the ground-based images can be attributed to the different surface brightness sensitivities of the two sets of images, and also in part to the differences in the methods applied to excise foreground and background objects. It is interesting to note that Roychowdhury et al. (2010) suggest that the gaseous disks of faint dwarf irregular galaxies are quite thick, with a mean intrinsic axial ratio $\mathrm{q}_{0} \simeq$ 0.57. The HST-based inclination of UGC 8245 reaches $90^{\circ}$ when $\mathrm{q}_{0}>0.48$; if $\mathrm{q}_{0}=0.57$ then the HST-based inclination of UGC 11411 increases to $42^{\circ}$. We note that for UGC 8245, which is effectively edge-on, the adopted inclination makes very little difference in the determination of the rotation velocities in $\S 3.3$, the effect is more significant for UGC 11411.

\subsection{Rotational Dynamics of UGC11411 and UGC 8245}

Given the small number of synthesized beams subtending each galaxy, we are unable to fit detailed two or three-dimensional models to derive their rotational dynamics. We therefore apply the techniques presented in McNichols et al. (2016) to create the position-velocity slices shown in Figures 1(c) and 2(c). The position angle of each slice is determined iteratively by maximizing the 
projected velocity along the kinematic major axis; this position angle is checked by examining the position angle of the isovelocity contours at the systemic velocity of each galaxy and then rotating each by $+90^{\circ}$. The central position of each slice is at the midpoint of the isovelocity contour at systemic velocity. Each position-velocity slice is 5 pixels wide and thus wider than the HI beam minor axis for each galaxy.

For UGC 11411, the position-velocity slice is centered at $(\alpha, \delta)=\left(19^{\mathrm{h}} 08^{\mathrm{m}} 42.5^{\mathrm{s}},+70^{\circ} 17^{\prime} 05.7^{\prime \prime}\right)$, following a position angle of $135^{\circ}$ measured east of north. As shown in Figure 1(c), the projected rotation of the galaxy shows the characteristic solid-body signature that is typical of low-mass galaxies (e.g., McNichols et al. 2016). We use the $4 \sigma$ contours in Figure 1 to define the total projected velocity $\left(\sim 87 \mathrm{~km} \mathrm{~s}^{-1}\right)$ and angular extent $\left( \pm 60^{\prime \prime}\right.$ from the dynamical center) of the gas. Correcting for inclination (see discussion above) and assuming symmetric projected rotation about the dynamical center at \pm 43.5 $\mathrm{km} \mathrm{s}^{-1}$, the corresponding dynamical mass estimates are $\mathrm{M}_{\mathrm{dyn}}=2.3 \times 10^{9} \mathrm{M}_{\odot}\left(i=37^{\circ}\right)$ and $\mathrm{M}_{\mathrm{dyn}}=1.4 \times 10^{9} \mathrm{M}_{\odot}$ $\left(i=52^{\circ}\right)$.

The major-axis position-velocity slice for UGC 8245 shows similar solid-body rotation characteristics as that for UGC 11411, although with a smaller projected velocity. As Figure2 shows, within the $4 \sigma$ contours, the total projected velocity is $\sim 44 \mathrm{~km} \mathrm{~s}^{-1}$ spanning an angular offset of $\pm 60^{\prime \prime}$. Interestingly, this major-axis slice shows a significant angular offset at all velocities, with some low surface brightness gas on the positive-velocity side of the slice; we interpret these features further in $\S$ 4 Correcting for inclination (see discussion above), and again assuming symmetric rotation about the dynamical center with a total magnitude of $\pm 22 \mathrm{~km} \mathrm{~s}^{-1}$, the resulting dynamical mass estimates are $\mathrm{M}_{\mathrm{dyn}}=1.7 \times 10^{8} \mathrm{M}_{\odot}(i=$ $\left.75^{\circ}\right)$ and $\mathrm{M}_{\mathrm{dyn}}=1.5 \times 10^{8} \mathrm{M}_{\odot}\left(i=90^{\circ}\right)$.

It is important to note that in the absence of detailed three-dimensional modeling, the angular resolution of these data results in a significant uncertainty on the derived dynamical mass of each galaxy. Specifically, while Figures 1 and 2 show high significance $(4 \sigma)$ HI gas at angular offsets of $\pm 60^{\prime \prime}$, this is only marginally larger than the minor axis dimensions of the synthesized beams; we cannot rule out that beam smearing has artificially enlarged the angular offset from the minor axis beam size to the estimated $\pm 60^{\prime \prime}$ values. Since $\mathrm{M}_{\mathrm{dyn}}$ scales linearly with angular offset, the dynamical mass estimates should be considered uncertain at the $50 \%$ level; higher angular resolution imaging of each galaxy is warranted.

\section{DISCUSSION}

Our new HI observations reveal that UGC 8245 is only marginally dark matter dominated. Correcting the detected $1.4 \times 10^{7} \mathrm{M}_{\odot}$ of $\mathrm{HI}$ by a factor of 1.35 for helium and metals, the sum of the total gas mass and the stellar mass is $\sim 9.2 \times 10^{7} \mathrm{M}_{\odot}$. The total dynamical mass is $\sim 2$ times larger than the baryonic mass, regardless of which inclination value is adopted for the disk. The physical properties of UGC 8245 are very similar to those of the more massive SHIELD galaxies discussed in McNichols et al. (2016); these galaxies share similar HI and stellar masses, as well as similar $\mathrm{M}_{\mathrm{dyn}} / \mathrm{M}_{\text {bary }}$ ratios.

UGC 11411, on the other hand, is a more dark matter dominated system that is more massive than all of the
SHIELD galaxies discussed in McNichols et al. (2016). Using the larger value of inclination $\left(i=52^{\circ}\right)$, the total baryonic mass $\left(\mathrm{M}_{\text {bary }}=1.1 \times 10^{8} \mathrm{M}_{\odot}\right)$ is smaller than the dynamical mass by a factor of more than 10 . If the inclination is in fact lower $\left(i=37^{\circ}\right)$ then the dynamical mass exceeds the baryonic mass by a factor of 20 . Such a highly dark matter dominated halo is unusual amongst the population of local volume dwarf galaxies.

Both of the galaxies presented in this work show the characteristic signatures of solid-body rotational dynamics. Interestingly, these two galaxies probe the beginning of the transition regime from rotationally-dominated to pressure-dominated systems that is discussed in detail in McNichols et al. (2016). In UGC 11411 the rotational velocity is much larger than the velocity dispersion at all radii. In contrast, the major axis position-velocity slice of UGC 8245 shows both a smaller projected rotation but also a wider velocity dispersion at all slice positions (and radii). Using the detailed discussion in McNichols et al. (2016) as a guide, this is most easily interpreted as the signature of low projected rotation velocity superposed on the characteristic velocity dispersion of the HI gas. The low (inclination corrected) rotation velocity of UGC 8245 is approaching (but still above) the empirical limit at which current observations can no longer differentiate between pressure-dominated and rotation-dominated systems.

Each of these galaxies has a low (barycentric) recessional velocity, and yet each is located well outside the Local Group. The HST-based distance measurements are accurate at the $\sim 5 \%$ level, which allows the peculiar velocities to be fixed with an accuracy of $\sim 20 \mathrm{~km} \mathrm{~s}^{-1}$ $\left(\mathrm{V}_{\mathrm{pec}}=-114 \mathrm{kms}^{-1}\right.$ and $-167 \mathrm{~km} \mathrm{~s}^{-1}$ for UGC 11411 and UGC 8245, respectively). Each of the galaxies is relatively isolated, and thus the negative peculiar velocities are not likely to be caused by virial motions alone. Further, the negative peculiar velocities are not unique; in a wide region of sky ( 3000 square degrees) around the considered galaxies there are more than a dozen galaxies with similarly low velocities (including the two massive spirals M 101 and NGC 6946). UGC 11411 and UGC 8245 appear to be associated with a coherent flow of nearby galaxies situated at high supergalactic latitude.

The authors are grateful to the NRAO for making the "Observing for University Classes" program available to the astronomical and teaching communities. We thank Macalester College and the Metropolitan State University of Denver for support. I.K. is supported by the Russian Science Foundation grant No 14-12-00965. The authors thank the anonymous referee for a prompt and insightful report that helped to strengthen this manuscript.

VLA, HST, GALEX

\section{REFERENCES}

Begum, A., Chengalur, J. N., Karachentsev, I. D., Sharina, M. E., \& Kaisin, S. S. 2008, MNRAS, 386, 1667

Berg, D. A., Skillman, E. D., Marble, A. R., et al. 2012, ApJ, 754, 98

Cannon, J. M., Giovanelli, R., Haynes, M. P., et al. 2011, ApJ, 739, L22

Cannon, J. M., McNichols, A. T., Teich, Y. G., et al. 2016, AJ, 152, 202 
Cannon, J. M., \& Van Moorsel, G. A. 2017, American Astronomical Society Meeting Abstracts, 229, 336.06

Cook, D. O., Dale, D. A., Johnson, B. D., et al. 2014, MNRAS, 445, 899

Giovanelli, R., Haynes, M. P., Kent, B. R., et al. 2005, AJ, 130, 2598

Hagen, C., Cannon, J. M., Cave, I., et al. 2014, American Astronomical Society Meeting Abstracts \#223, 223, \#355.16

Hunter, D. A., Ficut-Vicas, D., Ashley, T., et al. 2012, AJ, 144, 134

Karachentsev, I. D., Makarov, D. I., \& Kaisina, E. I. 2013, AJ, 145,101

Karachentsev, I. D., \& Kaisina, E. I. 2013, AJ, 146, 46

Kirby, E. M., Koribalski, B., Jerjen, H., \& López-Sánchez, Á. 2012, MNRAS, 420, 2924

Lelli, F., Verheijen, M., \& Fraternali, F. 2014, A\&A, 566, A71
McNichols, A. T., Teich, Y. G., Nims, E., et al. 2016, ApJ, 832, 89 Meyer, M. J., Zwaan, M. A., Webster, R. L., et al. 2004, MNRAS, 350,1195

Ott, J., Stilp, A. M., Warren, S. R., et al. 2012, AJ, 144, 123

Roychowdhury, S., Chengalur, J. N., Begum, A., \& Karachentsev, I. D. 2010, MNRAS, 404, L60

Swaters, R. A., van Albada, T. S., van der Hulst, J. M., \& Sancisi, R. 2002, A\&A, 390, 829

Teich, Y. G., McNichols, A. T., Nims, E., et al. 2016, ApJ, 832, 85

Tully, R. B., Courtois, H. M., Dolphin, A. E., et al. 2013, AJ, 146, 86

Wang, J., Koribalski, B. S., Jarrett, T. H., et al. 2017, MNRAS, in press $(\mathrm{ArXiV} / 1708.02744)$ 